\title{
Single-Molecule Manipulation of Enzyme Conformational and Catalytic
}

\section{Dynamics}

\author{
Yufan He, Maolin Lu, Jin Cao, H. Peter Lu
}

Bowling Green State University, Center for Photochemical Sciences, Department of Chemistry, Bowling Green, Ohio, USA

Proteins often involve complex conformational fluctuations and molecular interactions in playing their biological functions in living cells. The new paradigm of the protein structure-function relationship is that the dynamics of protein structural fluctuations play critical roles in protein functions. In recent years, manipulating single-molecule protein conformations for changing, enhancing, or even creating protein functions has been intensively developed and demonstrated in studying protein dynamics. In characterizing protein function and conformation activities, single-molecule approaches are proved to be particularly powerful and informative beyond the conventional ensemble-averaged approaches. Furthermore, it has been theoretically suggested, for example, that an oscillating force applied to an enzyme at a comparable frequency of enzymatic reaction turnover rate changes the enzymatic reaction activities, which is due to force modification of the reaction pathway, potential surface, and enzymatic reaction intermediate state energy.

There are two essential approaches to studying single-molecule protein dynamics: (1) Observing without perturbation or minimum perturbation, and (2) actively manipulating the protein while observing the protein dynamics. In recent years, studying protein dynamics beyond the simply observing has becoming increasingly informative and technical accessible; however, significant technical obstacles have to be overcomes in order to fully realize the high promises of the new single-molecule approach. Nevertheless, there are at least three crucial technical challenges need to be met: (1) to be able to manipulating a specific and targeted protein conformational coordinate; (2) to pin point the AFM tip on the top of the target proteins distributed in $10^{-9}-$ $10^{-11} \mathrm{M}$ concentration, i.e., averaged distance from a single protein to another is about 1 micrometer or larger; and (3) to be able to collect FRET signal on top of a high light scattering background from the AFM tip.

In this talk, we will discuss our recent work on combining atomic force microscopy (AFM) force manipulation and single-molecule fluorescence resonant energy transfer (FRET) spectroscopic imaging. [1,2] By residue-specific tethering of the protein to a microscopic cover slip, we achieved AFM tip manipulating a specific conformation coordinate at a specific residue of protein structure. Furthermore, using pulse laser excitation, we were able to monitoring the single-molecule FRET donor fluorescence lifetime to probe the FRET efficiency fluctuation associated with the protein conformational dynamics. By measuring lifetime based FRET changes rather than the intensity based FRET changes, we avoided the complications of picking up the FRET changes from a high light scattering background. 
We report our new technical approach of an AFM-FRET ultramicroscopy capable of simultaneous measurements of single-molecule force spectroscopy and FRET spectroscopy for a targeted single-molecule protein molecule. By recording and analyzing single-molecule FRET time trajectories of a Cy3-Cy5 labeled kinase protein and correlated force spectroscopy on the same molecule, we have demonstrated the experimental approach of simultaneous single-molecule spectroscopic measurements of protein conformational changes under AFM tip force manipulations. This is a significant advancement as the biological molecule structure and function relationship can now be studied in an actively controlled experiment for any given targeted single protein molecules.

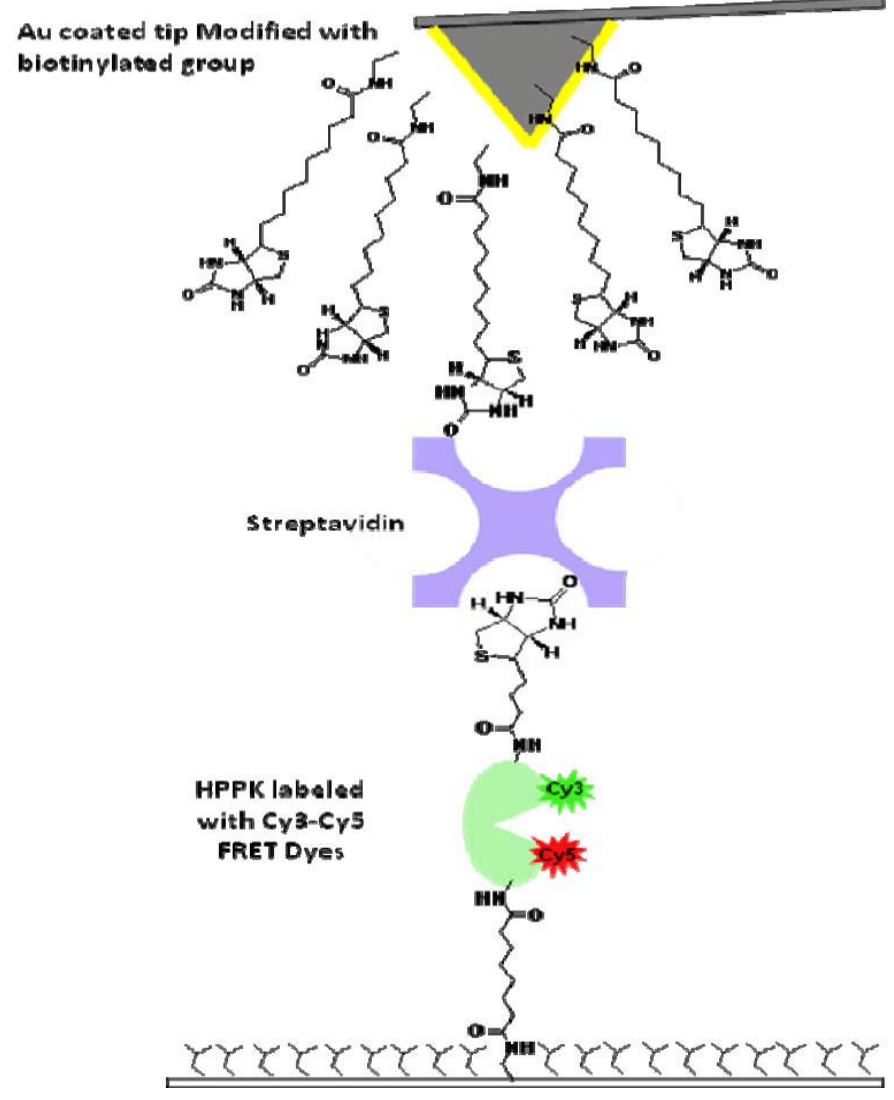

Figure 1. Single-molecule AFM-FRET ultra microscopy, a schematic diagram of one FRET dyes (Donor-Acceptor: Cy3-Cy5) pair labeled HPPK (a kinase) molecule tethered between a glass cover-slip surface and a handle (biotin group plus streptavidin), and a biotin group modified AFM tip. Single-molecule fluorescence photon counting images of the donor (Cy3) and accepter (Cy5) are obtained by donor-acceptor two channel microscopic imaging.

Acknowledgements:

This work is supported by the Basic Material Science program of the Army Research Office, and it is also supported in part by NIH NIGMS. We acknowledge stimulating discussions with Professor Honggao Yan of Michigan State University, and their kind offering with FRET labeled HPPK protein samples.

References:

1. Yufan He, Maolin Lu, Jin Cao, H. Peter Lu, "Manipulating Protein Conformations by Single-Molecule AFM-FRET Nanoscopy," ACS Nano, 6, 1221-1229 (2012).

2. Yufan He, Maolin Lu, H. Peter Lu, "Single-Molecule Photon Stamping FRET Spectroscopy Study of Enzymatic Conformational Dynamics," Phys. Chem. Chem. Phys., 15, 770-775 (2013) (Cover page). 